\title{
Examining the Role of Metabolites in Bioequivalence Assessment
}

\author{
Vangelis Karalis ${ }^{1}$ and Panos Macheras ${ }^{2}$ \\ ${ }^{1}$ Thriassio General Hospital of Elefsina, Greece \\ ${ }^{2}$ Faculty of Pharmacy, University of Athens \\ Received, March 5, 2010; Revised, May 24, 2010; Accepted, June 2, 2010; Published, June 10, 2010.
}

\begin{abstract}
Purpsoe. Investigate the role of metabolites in bioequivalence (BE) assessment. Methods. Sets of ordinary differential equations are used to generate concentration - time data for both parent drug (P) and metabolite (M). The calculations include 24 subjects, two different formulations (Test, Reference), and a range of Test/Reference ratios for the fraction of dose absorbed and the rate of absorption. A summarized view of these results is made through the construction of three dimensional power curves. The criteria for the choice of the preferred analyte $(\mathrm{P}$ or $\mathrm{M})$ are based on a sensitivity analysis of the bioequivalence measure $\left(A U C, C_{\max }\right)$. The latter depends on the relative ability of $\mathrm{P}$ and $\mathrm{M}$ to reflect better the changes of the pharmacokinetic parameters and variability. Results. The different sensitivity properties of $\mathrm{P}$ and $\mathrm{M}$ were reflected on the power curves. For $A U C$, the performance of metabolite is very similar to that of the parent drug for all scenarios and models examined. A more complex behaviour is evident for $C_{\max }$. In most of these cases, metabolite data show higher permissiveness in the percentages of acceptance. This attribute is more evident when $\mathrm{P}$ exhibits high elimination rate and/or the formation of $\mathrm{M}$ occurs rapidly. When the Test and Reference products have similar absorption profiles, metabolite data are preferable for the determination of bioequivalence. Parent drug has the advantage for detecting better the differences in the absorption rate of two drugs. The latter is counterbalanced by the increased sensitivity of $\mathrm{P}$ data to the variability of the data. Conclusions. Both parent drug and metabolite share the same ability to declare BE when AUC is used as a bioequivalence measure. In case of $C_{\max }$, metabolite data exhibit better performance when the $\mathrm{T}$ and $\mathrm{R}$ products are truly bioequivalent or the two formulations differ in their extent of absorption. For the pharmacokinetic scenarios studied, parent drug data were found to be more sensitive to detect differences in the rate of absorption. However, in such cases, their information is much influenced by the increased variability.
\end{abstract}

\section{INTRODUCTION}

Classically, bioequivalence (BE) assessment relies on the concept of average BE (1). Two drug products, a generic (Test, $\mathrm{T}$ ) versus a brand name product (Reference, $\mathrm{R}$ ) are considered to be bioequivalent if the calculated $90 \%$ confidence interval (CI) for the ratio of their mean measures of bioavailability is between predefined $\mathrm{BE}$ limits (1). In most of the cases, bioequivalence studies are carried out focusing only on the measurement of the parent drug $(\mathrm{P})$. Eventhough, the role of metabolites (M) in bioequivalence assessment has been the subject of many discussions, it still remains a controversial issue (2-6). The basic argument in favor of the use of the parent compound for $\mathrm{BE}$ assessment relies on the fact that the concentration $(C)$ - time $(t)$ profile of $\mathrm{P}$ is more sensitive to detect differences in formulation performance than $\mathrm{M}(5,6)$.

When the administered drug is either not metabolized or is the only active substance, the regulatory authorities recommend the use of the parent drug for BE assessment (7-9). On the other hand, the use of metabolites in BE studies cannot be disregarded since the latter reflects better drug distribution, elimination, and its formation through the parent drug (7). Nevertheless, there are situations where either both parent drug and metabolite data should be measured, or even the use of solely the metabolite data is preferable. Such situations include the cases where: a) the parent drug levels in biological fluids are too low to allow an accurate analytical measurement, b) parent drug is unstable in the biological matrix, c) $\mathrm{P}$ is an inactive prodrug which is transformed invivo into the active metabolite, $\mathrm{d}$ ) the formation of $\mathrm{M}$ occurs rapidly, and e) the metabolite contributes significantly to the net activity and the underlying pharmacokinetic (PK) system is nonlinear $(5,7,8,10)$.

Corresponding Author: Dr. Vangelis Karalis, Thriassio General Hospital of Elefsina, Elefsina, 19018, Attica, Greece, E-mail: vkaralis@pharm.uoa.gr 
Another option was to measure both $\mathrm{P}$ and $\mathrm{M}$ and use their sum as a measure of bioequivalence. However, it has been demonstrated that the application of the $0.80-1.25 \mathrm{BE}$ limits to the sum of $\mathrm{P}$ and $\mathrm{M}$ may result in misleading results (11).

The approaches used to investigate the role of metabolites in BE assessment can roughly be divided into two categories. According to the first approach, actual BE studies are being analyzed aiming to give evidence for the relative performance of the parent drug and the metabolite (12-20). A drawback of this method arises from the fact that the true condition is always unknown i.e., whether the two drug products are truly bioequivalent or not. The second methodology relies on the generation of simulated data. In this context, several studies have been published (3, 21-27). This approach offers the advantages of knowing always the correct answer and additionally, allows one to make inferences about several different types of parent drug and metabolite kinetics.

In other words, selecting the preferred analyte in $\mathrm{BE}$ assessment, relies on the fact that this moiety offers an advantage for the determination of bioequivalence. A possible advantage in $\mathrm{BE}$ assessment could be the ease of analytical measurement of this analyte (e.g., higher plasma concentration values or chemical stability), the relative contribution of this moiety to the net activity/toxicity, the measurement of this moiety reflects better the differences in the formulation performance etc.

In this study, the criteria for defining the "preferred analyte" were solely based on a bioequivalence context. Since, the bioequivalence decision depends on the ability of the measured moiety to identify differences in the responses between the $\mathrm{T}$ and $\mathrm{R}$ formulation, the analyte of choice would be the one which reflects "better" the differences in the extent $(F)$ and the rate $\left(K_{a}\right)$ of absorption. The term "better" simply means that the analyte of choice would be the one which carries the information of bioequivalence with a) higher sensitivity to detect differences in $F$ and $K_{a}$ ratios between the $\mathrm{T}$ and $\mathrm{R}$ formulations, and $\mathrm{b}$ ) lower variability (noise) of this response.

In this analysis, the role of metabolites in $\mathrm{BE}$ assessment was investigated using simulated bioequivalence studies. This task was implemented by generating data for a variety of pharmacokinetic models and scenarios, in order to allow inferences to as many as possible different conditions. The simulated data were then used to simulate the conditions of bioequivalence trials.
In order to allow the application of both simple and more complex models, the mathematical description of the models was made through the use of ordinary differential equations.

\section{METHODS}

\section{Pharmacokinetic Simulations}

\section{Pharmacokinetic Models}

In order to compare the performance of the parent drug and the metabolite, various conditions were included in the simulations. One-and twocompartment models were assumed for either $\mathrm{P}$ or $\mathrm{M}$; a schematic representation of the pharmacokinetic models used in this study is depicted in Figure 1. Models A-C (Figure 1) correspond to the situations where the parent drug obeys first order absorption kinetics without first pass effect, whereas in models from D to I (Figure 1) the parent drug undergoes metabolism during its absorption.

Formation of the metabolite is assumed to take place in the central compartment from the parent drug following first order kinetics $(21,22)$. In cases where first pass effect co-exists, metabolite formation also occurs in the GI lumen. In the latter situation, Michaelis-Menten kinetics is used to describe the metabolite formation. It should be highlighted that only one metabolite is considered in the simulations. In addition, no hypotheses for pharmacological activities are made, since the aim of this study is to provide an assessment of the relative performance of $\mathrm{P}$ and $M$ relying only on a bioequivalence context.

In case of two compartment models, drug transfer from the central to the peripheral compartment - and vice versa - is assumed to follow first order kinetics. In all cases, elimination of both $\mathrm{P}$ and $\mathrm{M}$ compounds takes place in the central compartment through first order kinetics. For all pharmacokinetic models considered in this analysis, only a first generation metabolite is considered to be formed from the parent drug.

Based on mass balance principles, each pharmacokinetic model is described with a system of ordinary differential equations which were solved numerically. Two representative examples of systems of differential equations are shown in Appendix. These examples correspond to the simplest case of one-compartment model and to a more complex situation where parent drug obeys two-compartment disposition kinetics and undergoes first-pass metabolism. 


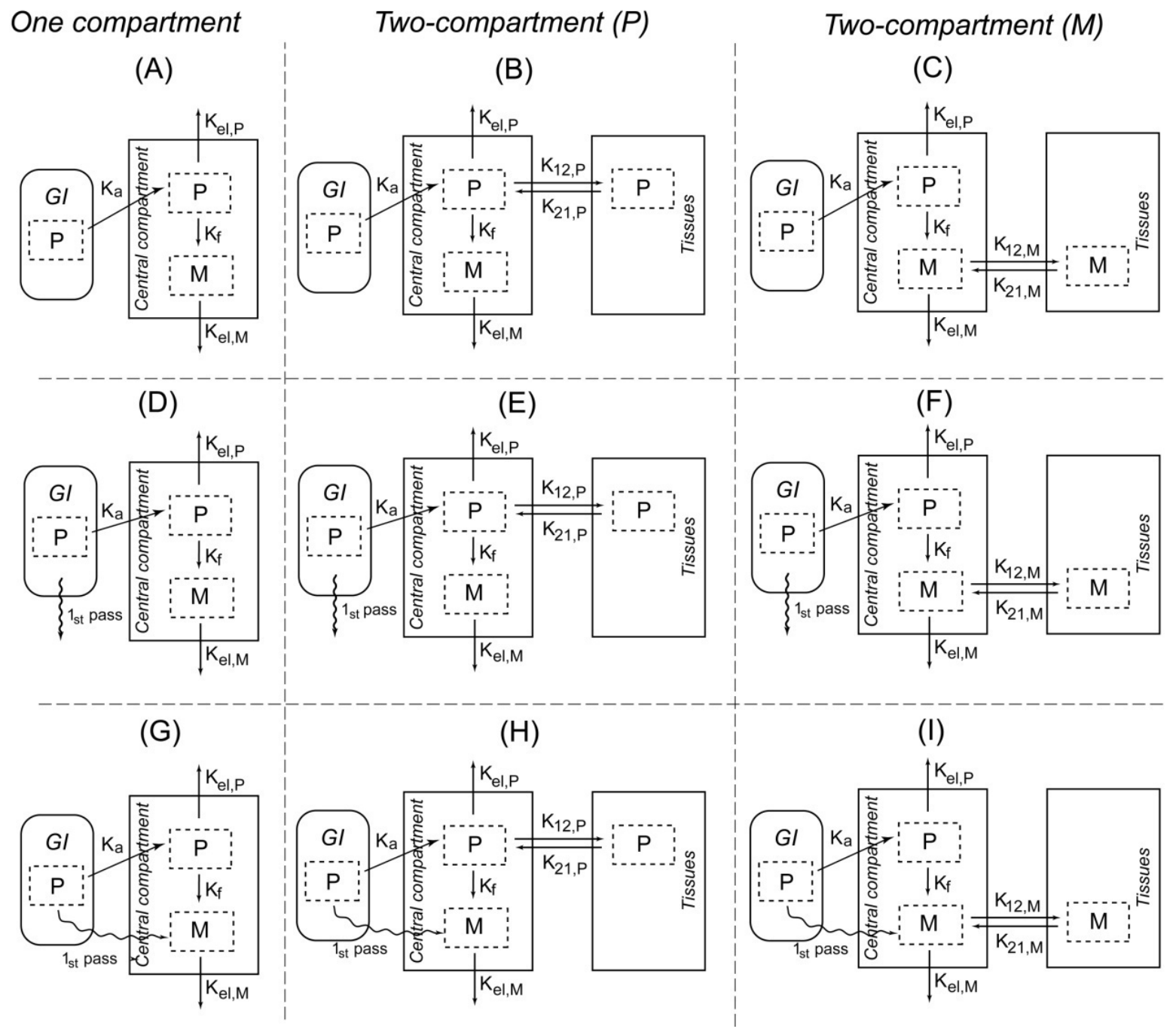

Figure 1. Pharmacokinetic models for the simulation of parent drug (P) and metabolite (M) disposition kinetics. Oneand two-compartment models are assumed for $\mathrm{P}$ and $\mathrm{M}$ for each of the following cases: (A-C) First-order absorption kinetics, (D-F) First-order absorption of $\mathrm{P}$ and first-pass effect according to Michaelis-Menten kinetics leading to metabolite(s) other from the one measured, (G-I) First-order absorption of $\mathrm{P}$ and first-pass effect which leads to concomitant formation of the metabolite under study. Key: $F$, the bioavailable fraction of dose; GI, the gastrointestinal tube; $K_{a}$, absorption rate constant, $K_{f}$, first-order formation rate constant of the metabolite; $K_{e l, i}$, elimination rate constant; $K_{12, i}$ central compartment to tissue rate constant; $K_{21, i}$ tissue to central compartment rate constant. In all cases the index $i$ refers to either $\mathrm{P}$ or $\mathrm{M}$.

\section{Pharmacokinetic Scenarios}

The relative performance of the parent drug versus metabolite was evaluated using several pharmacokinetic conditions. In order to generate the appropriate concentration-time profiles for $\mathrm{P}$ and $M$, certain values were assigned to the parameters of the pharmacokinetic models shown in Figure 1. Therefore, depending on the pharmacokinetic parameters' values several pharmacokinetic scenarios were developed. The pharmacokinetic scenarios along with the average values of the parameters used in the analysis are listed in Table 1. These scenarios were selected to give an insight into conditions that include: i) typical drug kinetics based on the one- or twocompartment model, ii) rapid or slow absorption of the parent drug, iii) high or low elimination rate of the parent drug and the metabolite, iv) fast or slow formation of the metabolite, and v) high or low first-pass effect of the parent drug. 
Table 1. Parameter values for the pharmacokinetic scenarios used in simulations of both parent drug $(\mathrm{P})$ and metabolite $(\mathrm{M})$.

\begin{tabular}{|c|c|c|c|c|c|c|c|c|c|c|c|c|c|c|c|}
\hline \multirow{3}{*}{ Scenario } & \multirow{3}{*}{ Description } & \multicolumn{14}{|c|}{ Parameters } \\
\hline & & \multirow{2}{*}{$\begin{array}{c}K_{a} \\
\left(\mathbf{h}^{-1}\right)\end{array}$} & \multirow{2}{*}{$K_{e l, P}\left(h^{-}\right.$} & \multirow{2}{*}{$\begin{array}{l}K_{e l, M} \\
\left(\mathbf{h}^{-1}\right)\end{array}$} & \multirow{2}{*}{$\begin{array}{c}K_{f} \\
\left(\mathbf{h}^{-1}\right)\end{array}$} & \multirow{2}{*}{$\begin{array}{l}V_{1, P} \\
(\mathrm{~L})\end{array}$} & \multirow{2}{*}{$\begin{array}{l}V_{1, M} \\
(\mathrm{~L})\end{array}$} & \multirow{2}{*}{$\begin{array}{c}M M_{0} \\
(\mathrm{mg} / \mathrm{h})\end{array}$} & \multirow{2}{*}{$\begin{array}{c}M M_{50} \\
(\mathrm{mg} / \mathrm{L})\end{array}$} & \multicolumn{3}{|c|}{$\boldsymbol{K}_{\mathbf{1 2 , i}}\left(\mathbf{h}^{-\mathbf{1}}\right)^{1,2}$} & \multicolumn{3}{|c|}{$\boldsymbol{K}_{21, \mathbf{i}}\left(\mathbf{h}^{-\mathbf{1}}\right)^{1,2}$} \\
\hline & & & & & & & & & & $a$ & $b$ & $c$ & $a$ & $b$ & $c$ \\
\hline \multicolumn{16}{|c|}{ First order absorption (Figure 1: A-C) } \\
\hline$S 1$ & Typical model & 0.50 & 0.15 & 0.20 & 0.25 & \multirow{8}{*}{10.0} & \multirow{8}{*}{10.0} & - & - & \multirow{8}{*}{0.15} & \multirow{8}{*}{0.80} & \multirow{8}{*}{0.01} & \multirow{8}{*}{0.10} & \multirow{8}{*}{0.01} & \multirow{8}{*}{0.01} \\
\hline S2 & Rapid absorption of $\mathrm{P}$ & 1.20 & 0.15 & 0.20 & 0.25 & & & - & - & & & & & & \\
\hline S3 & Slow absorption of $\mathrm{P}$ & 0.20 & 0.15 & 0.20 & 0.25 & & & - & - & & & & & & \\
\hline S5 & Low elimination rate of $P$ & 0.50 & 0.01 & 0.20 & 0.25 & & & - & - & & & & & & \\
\hline$S 6$ & High elimination rate of $\mathrm{M}$ & 0.50 & 0.15 & 1.50 & 0.25 & & & - & - & & & & & & \\
\hline S7 & Low elimination rate of $\mathrm{M}$ & 0.50 & 0.15 & 0.05 & 0.25 & & & - & - & & & & & & \\
\hline S8 & High metabolite formation rate & 0.50 & 0.15 & 0.20 & 2.00 & & & - & - & & & & & & \\
\hline S9 & Low metabolite formation rate & 0.50 & 0.15 & 0.20 & 0.02 & & & - & - & & & & & & \\
\hline \multicolumn{16}{|c|}{ First order absorption and first-pass effect (Figure. 1: D-I) } \\
\hline S11 & $P$ undergoes extensive $1^{\text {st }}$ pass effect & 0.50 & 0.15 & 0.20 & 0.25 & 10.0 & 10.0 & 20.0 & 30.0 & 0.15 & 0.80 & 0.01 & 0.10 & 0.01 & 0.01 \\
\hline$S 12$ & $\begin{array}{l}\mathrm{P} \text { is transformed into } \mathrm{M} \text { under } 1^{\text {st }} \text { pass } \\
\text { effect }\end{array}$ & 0.50 & 0.15 & 0.20 & 0.25 & 10.0 & 10.0 & 1.0 & 30.0 & 0.15 & 0.80 & 0.01 & 0.10 & 0.01 & 0.01 \\
\hline
\end{tabular}

${ }^{1} \mathrm{~K}_{12, \mathrm{i}}$ and $\mathrm{K}_{21, \mathrm{i}}$ values do not exist for the one-compartment model.

${ }^{2}$ When parent drug obeys two-compartment disposition kinetics $i=P$, while for the two-compartment model of the metabolite $i=M$.

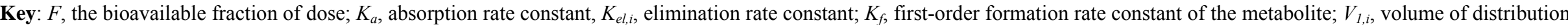

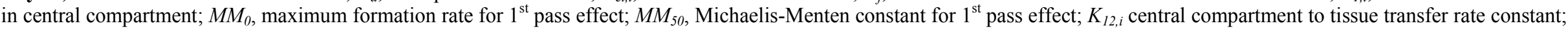
$K_{21, i}$ tissue to central compartment transfer rate constant. In all cases the index $i$ refers to either P or M. 
Depending on the relative transfer rate between the central compartment and the tissues, each of the two-compartment models for parent drug $(2 C P)$ or metabolite $(2 C M)$ was further subdivided into three categories $(a, b, c)$, Subcategory $a$ includes the cases where both the transfer from the central compartment to the tissues $\left(K_{12, P}\right.$ or $\left.K_{12, M}\right)$, as well as the comeback $\left(K_{21, P}\right.$ or $\left.K_{21, M}\right)$, are expressed with similar rates of an intermediate degree. The other two subcategories $(b, c)$ refer to the cases of: i) rapid transfer to tissues with slow return from the periphery (type $b$ ), and ii) slow distribution to the tissues with rapid arrival at the central compartment (type $c$ ). It should be mentioned that there was no reason to apply concomitantly high $K_{12, P}\left(\right.$ or $\left.K_{12, M}\right)$ and $K_{21, P}\left(\right.$ or $\left.K_{21, M}\right)$ values, since this situation results in a rapid equilibrium between the central compartment and the periphery, a case which performs kinetically as an one-compartment model. For the same reason, there was no reason to use low $K_{12, P}$ (or $K_{12, M}$ ) values with rapid arrival rate at the central compartment (i.e., high $K_{21, P}$ or $K_{21, M}$ ).

\section{Simulated Bioequivalence Trials}

Two-period, two-treatment (Test versus Reference), crossover bioequivalence studies, with equal number of subjects in each sequence, were simulated using the classic $(0.80-1.25) \mathrm{BE}$ limits. A number of 24 subjects were assumed to participate in each trial. The subjects were randomly divided into two sequences of drug administration with equal number of subjects in each sequence.

Inter- and intra-individual variability were considered for all pharmacokinetic parameters. Uniform distribution was used for the random generation of the fraction of dose absorbed, while for the remaining parameters, inter- and intraindividual variability was added assuming lognormal distribution. In the latter cases, the standard deviations $\left(\sigma_{i}\right)$ of the logarithmically transformed parameters were calculated from the preset variability $\left(\mathrm{CV}_{i}\right)$ using the following expression: $\sigma_{i}=\sqrt{\ln \left(1+C V_{i}^{2}\right)}$ where the index $i$ refers to each parameter separately. The coefficient of variation values for the inter- and intra-individual variability of $F, K_{a}$, volumes of drug distribution, and the rest rate constants was set equal to $20 \%, 30 \%, 2 \%$, and $5 \%$, respectively (see Table 2). Presumably, these different variability values lead to different intra-individual variability $\left(C V_{W}\right)$ values for $A U C$ and $C_{\max }$ estimates.

According to the simulated $C-t$ profiles of parent drug and metabolite, appropriate sampling schemes were considered. These sampling schemes were selected to provide an adequate number of observations in the ascending limb of the curves, as well as to be large enough so as to describe sufficiently the total $C$ - $t$ profile (see Table 3). In order to allow a fair treatment of all PK scenarios, the utilized experimental design comprised the same observations at early timepoints, while more sampling points were added for long time periods depending on the scenario (see Table 4).

There was also the need for the estimation of the bioavailability measures. $C_{\max }$ was simply identified as the highest recorded concentration of a given concentration - time profile. Area under the concentration - time curve was estimated based on the simulated $C$ - $t$ profile and the selected concentration values from the appropriate sampling scheme. $A U C$ was calculated from time zero to the last generated concentration time point $\left(C_{\text {last }}\right)$ using the linear trapezoidal method.

A variety of bioavailable fractions and absorption rate constants were assumed depending on our intention to place more emphasis on the extent or the rate of absorption, respectively. These relative $F$ and $K_{a}$ values were gradually increased from 1.00 to 1.30 with a step of 0.05 .

In all cases, an arbitrary dose of $100 \mathrm{mg}$ of drug was assumed to be administered. Under each condition, five thousand $(5,000)$ simulated $\mathrm{BE}$ trials were performed and the percentage of simulated studies, in which BE is accepted, was recorded. Bioequivalence was declared if the $90 \%$ confidence interval around the ratio of the estimated geometric means for the two drug products was between the BE limits (28). The whole programming work was implemented by developing a computer program in FORTRAN. Some subroutines, such as the uniform, the lognormal random generator, and the linear regression analysis routines, were borrowed from the IMSL libraries of the Compaq Visual Fortran v.6.5 package. Other subroutines, such as those used for the numerical solution of the system of ordinary differential equations were obtained from Netlib (http://www.netlib.org). All program subroutines and calculations were validated, prior to their application, so as to provide accurate results. 


\begin{tabular}{|c|c|c|}
\hline Parameter & Inter-individual variability & Intra-individual variability $\left(C V_{W}\right)$ \\
\hline$K_{a}($ Test $)$ & $30 \%$ & - \\
\hline$K_{a}($ Reference $)$ & $30 \%$ & - \\
\hline$K_{e l, P}$ & $5 \%$ & $5 \%$ \\
\hline$K_{e l, M}$ & $5 \%$ & $5 \%$ \\
\hline$K_{f}$ & $5 \%$ & $5 \%$ \\
\hline$V_{l, P}$ & $2 \%$ & $5 \%$ \\
\hline$V_{l, M}$ & $2 \%$ & $5 \%$ \\
\hline$M M_{0}$ & $5 \%$ & $5 \%$ \\
\hline$M M_{50}$ & $5 \%$ & $5 \%$ \\
\hline$K_{12, M}$ & $5 \%$ & $5 \%$ \\
\hline$K_{2 l, M}$ & $5 \%$ & $5 \%$ \\
\hline$K_{12, P}$ & $5 \%$ & $5 \%$ \\
\hline$K_{21, P}$ & $5 \%$ & $5 \%$ \\
\hline
\end{tabular}

Table 3. Sampling schemes used in simulation studies. For each pharmacokinetic scenario the appropriate sampling schedule was selected.

\begin{tabular}{ccc}
\hline Name of sampling scheme & \multicolumn{1}{c}{ Sampling points (hrs) } & Number of points \\
\hline SSC1 & $0,1,2,3,4,6,8,12,15,18$ & 10 \\
SSC2 & $0,1,2,3,4,6,8,12,15,18,24$ & 11 \\
SSC3 & $0,1,2,3,4,6,8,12,15,18,24,36$ & 12 \\
SSC4 & $0,1,2,3,4,6,8,12,15,18,24,36,48$ & 13 \\
\hline
\end{tabular}

\section{Assessment of the Performance}

\section{Modified Power Curves}

The results of the simulated BE trials were presented in the form of power curves. In order to represent the large amount of information derived from the abovementioned analysis in a compressed form, three dimensional power curves were constructed. The $z$-axis of these modified power curves refers to the difference, Diff(\%), between the power of parent drug and metabolite $($ Diff $\%=\%$ Power of $\mathrm{P}-\%$ Power of $\mathrm{M})$. Presumably, a negative Diff\% value implies that $\mathrm{P}$ is less permissive than M. The other two axes ( $x$ and $y$ ) correspond to the PK scenario and the ratio of $F_{T} / F_{R}$ (fraction of dose absorbed of $\mathrm{T}$ over R) or $K_{a T} / K_{a R}$ (absorption rate constant of $\mathrm{T}$ over R), respectively. This kind of illustration was adopted since it allows a pictorial view of the overall comparative performance of the two moieties ( $\mathrm{P}$ and $\mathrm{M})$.

\section{Sensitivity}

The decision of bioequivalence relies on the ability (sensitivity) of the measured moiety to identify differences in the responses between the $\mathrm{T}$ and $\mathrm{R}$ formulations. This decision is mathematically expressed with Equation 1:

$\ln (0.80) \leq\left(m_{T}-m_{R}\right) \pm t_{0.05, N-2} M S E \sqrt{\frac{2}{N}} \leq \ln (1.25)$ where: $m_{T}-m_{R}$ is the difference between the estimated logarithmic means (i.e., the GMR ratio), $N$ is the number of subjects participating in the BE study, $t$ is the t-student statistic at the probability of $a=0.05$ with $N-2$ degrees of freedom, and MSE is the Mean Square Error calculated from ANOVA. 
Table 4. Pharmacokinetic scenarios and the corresponding sampling schemes used in the simulations. The description of each sampling scheme is listed in Table 3.

\begin{tabular}{|c|c|c|c|c|c|c|c|}
\hline \multirow{3}{*}{ Scenario } & \multicolumn{7}{|c|}{ Sampling scheme } \\
\hline & \multirow[t]{2}{*}{ One-compartment model } & \multicolumn{3}{|c|}{ Two-compartment model (Parent drug, P) } & \multicolumn{3}{|c|}{ Two-compartment model (Metabolite, M) } \\
\hline & & $a$ & b & $c$ & $a$ & $\boldsymbol{b}$ & $C$ \\
\hline$S 1$ & $S S C 2$ & $S S C 3$ & $S S C 2$ & $S S C 2$ & $S S C 3$ & SSC1 & $S S C 2$ \\
\hline$S 2$ & $S S C 2$ & $S S C 2$ & $S S C 2$ & $S S C 2$ & SSC3 & SSC1 & $S S C 2$ \\
\hline$S 3$ & $S S C 3$ & $S S C 3$ & $S S C 3$ & $S S C 3$ & SSC3 & SSC3 & SSC3 \\
\hline$S 4$ & $S S C 2$ & $S S C 2$ & $S S C 2$ & $S S C 2$ & $S S C 3$ & SSC1 & $S S C 2$ \\
\hline S5 & SSC3 & SSC3 & SSC3 & SSC3 & SSC3 & SSC3 & SSC3 \\
\hline S6 & SSC2 & SSC2 & SSC1 & SSC1 & SSC2 & SSC1 & $S S C 2$ \\
\hline$S 7$ & SSC4 & SSC4 & SSC4 & SSC4 & SSC4 & SSC1 & SSC4 \\
\hline$S 8$ & $S S C 2$ & SSC2 & $S S C 2$ & SSC2 & SSC2 & SSC1 & $S S C 2$ \\
\hline$S 9$ & SSC3 & SSC3 & SSC3 & SSC3 & SSC3 & SSC3 & SSC3 \\
\hline$S 10$ & SSC2 & SSC3 & $S S C 2$ & SSC2 & SSC3 & SSC2 & $S S C 2$ \\
\hline$S 11$ & SSC2 & SSC3 & $S S C 2$ & $S S C 2$ & SSC3 & SSC1 & $S S C 2$ \\
\hline$S 12$ & $S S C 2$ & SSC3 & $S S C 2$ & $S S C 2$ & SSC3 & SSC1 & $S S C 2$ \\
\hline
\end{tabular}

In other words, "sensitivity" is a composite term (29) which is considered to express the two intrinsic properties of the measured analyte:

a. Sensitivity to $P K$ parameters. It simply means the ability of the moiety to transfer the underlying differences in extent and rate of absorption into changes of GMR values. In other words, sensitivity to changes reflects how sharp is the change of $G M R$ (for the measure under study e.g., $C_{\max }$ ) in response to relevant changes of the parameters responsible for that deviation. To this point, it is suggested that the $F_{T} / F_{R}$ or the $K_{a T} / K_{a R}$ ratio could serve as the underlying reason which cause the changes of GMR.

b. Sensitivity to error. This term represents the influence of the response due to error (i.e., intra-individual variability). Sensitivity to variability depends on intra-individual variability of the drug. According to Equation 1, sensitivity to error is expressed through the MSE term of ANOVA.

Therefore, the acceptance of BE becomes more strict when either "a" or "b" situations become more evident. In the light of information theory, the ideal situation would be the one where the "a" type of sensitivity is as much as higher, whereas the "b" type (or noise) gets the lowest possible value. In other words, the desired performance would be the one which exhibits: a) high percentage of acceptance when $G M R$ is equal to unity, namely, when the two drug products are truly bioequivalent, and b) low percentage of acceptance similar to the significance level $(\alpha=5 \%)$ when $G M R$ is equal to 1.25 (or 0.80 ).

In this study, sensitivity terms were quantified by applying the flowing 
procedure:

i) Sensitivity to $P K$ parameters. Plot the GMR values of the measure under study (e.g., $C_{\max }$ ) versus the relevant $K_{a T} / K_{a R}$ (or $F_{T} / F_{R}$ ) ratios for the parent drug. Apply linear regression to the pairs $\left(K_{a T} / K_{a R}, G M R\right)$ and estimate the slope. This procedure should be applied to the parent drug and the metabolite, separately. The relative sensitivity to changes of $\mathrm{P}$ over $\mathrm{M}$ is simply the ratio of the two slopes e.g., slope $_{P} /$ slope $_{M}$. A value equal to 1 simply implies that both $\mathrm{P}$ and $\mathrm{M}$ exhibit the same ability to perceive the differences in the changes of $K_{a T} / K_{a R}$. Besides, values higher (or lower) than unity, reflect a higher (or lower) sensitivity of the parent drug than the metabolite. For example, a value equal to 0.363 , implies that the metabolite data are less sensitive to detect differences in the changes of the $K_{a T} / K_{a R}$ ratio.

ii) The term sensitivity to variability is simply considered as the ratio (of metabolite versus parent drug) of the two residual variabilities, when the $K_{a T} / K_{a R}$ ratio is equal to 1.25 . In mathematical formalism, this is expressed as $C V_{W, M} / C V_{W, P}$. A value equal to unity, means that $\mathrm{P}$ and $\mathrm{M}$ are influenced in the same degree by variability, while values lower (or greater) than 1 imply that parent drug (or metabolite) is more vulnerable to noise.

The different sensitivity properties of parent drug and metabolite are reflected on the power curves. In other words, the percentages of acceptance represent the inherent properties for each moiety to demonstrate how sensitive is the $C-t$ profile of this moiety (P or M) to the specific conditions of the study. A more strict performance in the $\%$ acceptance values in power curves simply implies that the $\mathrm{P}$ or $\mathrm{M}$ data exhibit a higher sensitivity in the conditions of the study. Depending on the simulated PK scenario, different sensitivity estimates can be obtained for $A U C$ and $C_{\max } /$

\section{RESULTS}

\section{Area Under the Curve (AUC)}

Figure 2 illustrates the results for $A U C$ when both parent drug and metabolite obey onecompartment model disposition kinetics. Three dimensional power curves were constructed, where the difference in the percentages of acceptances between $\mathrm{P}$ and $\mathrm{M}$ is plotted against
PK scenario and $F_{T} / F_{R}$ ratios. In the majority of the scenarios examined for $A U C$, the probability of concluding bioequivalence is similar and independent from the PK scenario and the $F_{T} / F_{R}$ ratio. In other words, both parent drug and metabolite can lead to similar conclusions for bioequivalence, either when truly bioequivalent or bioinequivalent drug products are compared.

A similar to the abovementioned behaviour was observed when the parent drug or the metabolite follows two-compartment disposition kinetics (data available upon request). The probability of concluding bioequivalence using $A U C$ for the metabolite was almost the same with that of the parent drug, irrespective of the type of the pharmacokinetic scenario or the type of transfer $(a, b, c)$ between the central compartment and the tissues. The same conclusions were also obtained when the relative performance of $\mathrm{P}$ and $\mathrm{M}$ data were studied against a variety of $K_{a T} / K_{a R}$ values (data available upon request). Again, there was no difference in the performance for declaring $\mathrm{BE}$ between parent drug and metabolite.

\section{Peak Plasma Concentration $\left(C_{\max }\right)$}

Figure 3 shows the results for $C_{\max }$ when both $\mathrm{P}$ and $\mathrm{M}$ follow one-compartment kinetics and several ratios of $F_{T} / F_{R}$ are used. Twelve pharmacokinetic scenarios are presented where the $F_{T} / F_{R}$ ratio ranges from 1.00 to 1.30 . Visual inspection of Figure 3 reveals a more complex behaviour for $C_{\max }$. A common feature in Figure 3 is that in almost all cases the $C_{\max }$ values of the parent drug data are more sensitive than the relevant metabolite data. This is reflected on the higher percentages of $\mathrm{BE}$ acceptance for $\mathrm{M}$ data. The major discrepancy in the relative performance of $\mathrm{P}$ and $\mathrm{M}$ data is observed when $F_{T} / F_{R}$ gets close to unity, whereas as the $F_{T} / F_{R}$ ratio increases, the differences become smaller. Furthermore, there are certain situations according to which the metabolite data show much higher probability of declaring BE. This is predominant in cases of scenarios $S 4$ and $S 8$, which correspond to the conditions of high $\mathrm{P}$ elimination rate and high metabolite formation rate, respectively. Also, relatively higher percentages of acceptance for $\mathrm{M}$ are shown in scenarios $S 1, S 3, S 7, S 12$, and $S 10$ which refer to the conditions of a typical one-compartment model, slow absorption of $\mathrm{P}$, low elimination rate of $\mathrm{M}$, and intermediate degree of first-pass effect with or without concomitant formation of the $\mathrm{M}$, respectively (see Table 1). 


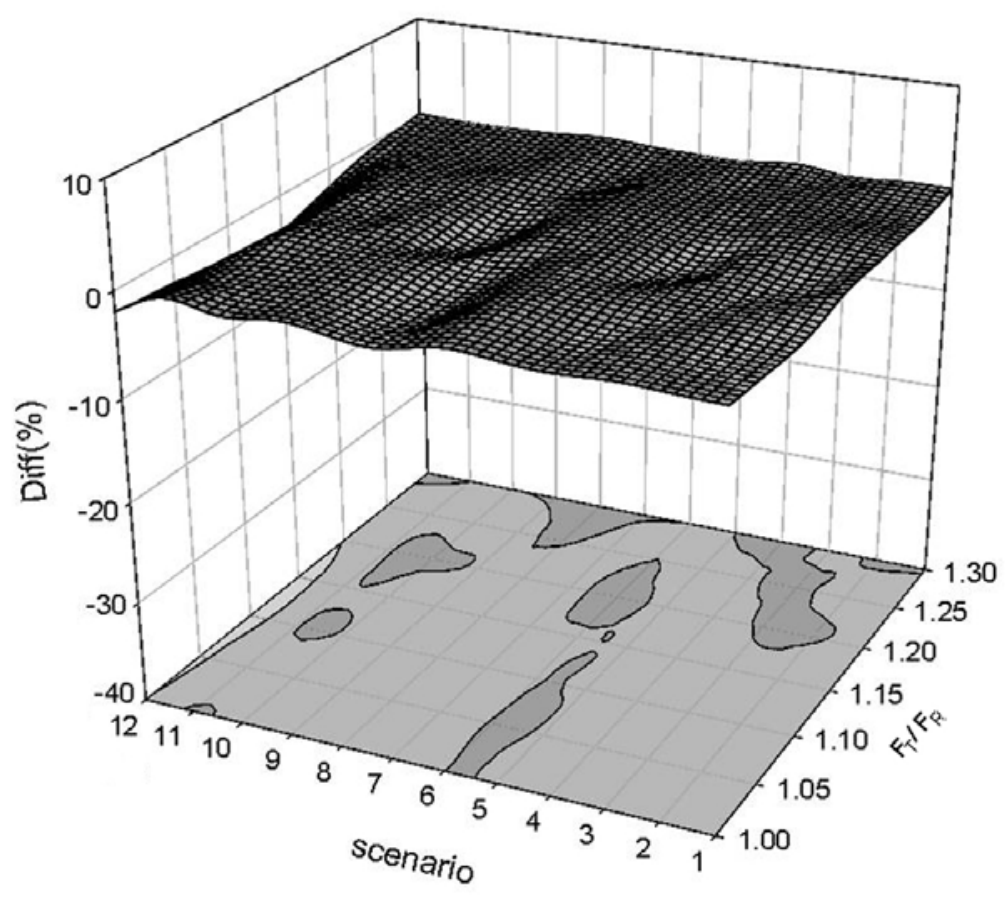

Figure 2. Three dimensional power curves illustrating the difference (Diff $\%$ ) in $\%$ acceptance of bioequivalence for $A U C$ between parent drug and metabolite at various scenarios and $F_{T} / F_{R}$ ratios in case of one-compartment model. Diff $\%$ is estimated by subtracting the $\%$ acceptance of metabolite from that of the parent drug. Under each condition, a number of 5,000 studies are simulated.

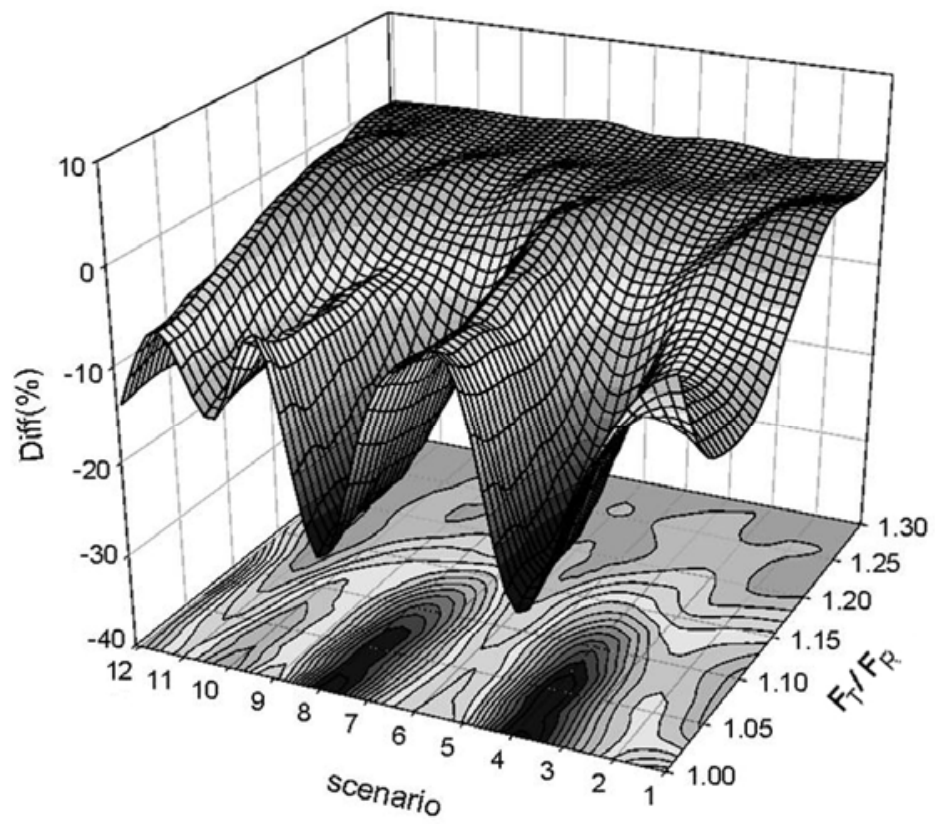

Figure 3. Three dimensional power curves illustrating the difference (Diff $\%)$ in $\%$ acceptance of bioequivalence for $C_{\max }$ between parent drug and metabolite at various scenarios and $F_{T} / F_{R}$ ratios in case of one-compartment model. $D i f f \%$ is estimated by subtracting the $\%$ acceptance of metabolite from that of the parent drug. Under each condition, a number of 5,000 studies are simulated. 
The three dimensional power curves for $C_{\max }$, when several $F_{T} / F_{R}$ ratios were used, are shown in Figure 4. The first (A1-A3) set of Figure 4 corresponds to the situation where parent drug obeys two-compartment disposition kinetics, whereas in the second set (B1-B3) the metabolite follows two-compartment kinetics and parent drug is confined only in the central compartment. All these plots reveal a complex behaviour for $C_{\max }$, similar to that described above for the onecompartment model (Figure 3). A common feature of all these plots is the higher percentages of acceptance when metabolite is used instead of the parent drug. Accordingly, this is more evident for $F_{T} / F_{R}$ values close to unity. The higher ability of metabolite data to declare bioequivalence is also more apparent in scenarios $S 4$ and $S 8$. Higher percentages of acceptance for $\mathrm{M}$ data, but to a lower degree, can also be observed in case of scenarios $S 1, S 3, S 7, S 10$, and $S 12$. It should be highlighted that these results are in full accordance with the results presented earlier for the one-compartment model for $C_{\max }$ (Figure 3).

The described behaviour of the parent drug for the $2 C P$ model is similar for all the three types $(a$, $b, c)$ of transfer between the central compartment and the tissues. However, the differences in the performance between $\mathrm{M}$ and $\mathrm{P}$ are more obvious in case of the $b$ type of transfer i.e., when the parent compound distributes rapidly to tissues and its arrival at the central compartment occurs with very slow rate. Furthermore, metabolite data in case of $b$ type of transfer were found to be more permissive even in cases of scenarios $S 5$ and $S 9$, which correspond to the conditions of low elimination rate for $\mathrm{P}$ and low formation rate for $\mathrm{M}$, respectively.

The three dimensional power curves illustrated in Figure 4 (B1-B3) reflect the performance of $\mathrm{P}$ and $\mathrm{M}$ when metabolite is assumed to obey twocompartment disposition kinetics. Overall, the results derived for the $2 C M$ model are in accordance with the findings presented earlier from the analysis of simulations using twocompartment model for the parent drug and the simplest one-compartment model kinetics (Figure 3). Nevertheless, visual inspection of Figures 4A2 and 4B2 reveals that in cases where the metabolite follows two-compartment model kinetics with the $b$ type of transfer, the discrepancy in the performance between $\mathrm{P}$ and $\mathrm{M}$ is now reduced (Figure 4B2).

Figure 5 represents the results for $C_{\max }$ when both $\mathrm{P}$ and $\mathrm{M}$ follow one-compartment kinetics for twelve PK scenarios and several values of the
$K_{a T} / K_{a R}$ ratio. It is evident that the plots depicted in Figure 5 correspond to the most complex behaviour presented so far in this work. Overall, the sensitivity of $C_{\max }$ is less for the metabolite than the parent drug. At $K_{a T} / K_{a R}$ value equal to unity, the major difference between $\mathrm{M}$ and $\mathrm{P}$ data is observed for scenarios $S 4, S 8$, and $S 12$. This finding is in accordance with that observed earlier for $C_{\max }$ when several $F_{T} / F_{R}$ ratios were studied (Figures 3 and 4). However, as $K_{a T} / K_{a R}$ values rise, the discrepancy in the behaviour of $\mathrm{M}$ and $\mathrm{P}$ data, instead of diminishing, is now becoming more evident. This attribute represents a major difference in the performance of $\mathrm{M}$ and $\mathrm{P}$ data when the latter studied in the light of changes of absorption rate. In other words, the absolute difference in the \% of acceptance of $\mathrm{P}$ and $\mathrm{M}$ is getting greater. Thus, for $K_{a T} / K_{a R}$ values close to 1.25 , a discrepancy in the performance of $\mathrm{M}$ and $\mathrm{P}$ data can be observed for almost all scenarios. However, even in these cases, the difference is greater for scenarios $S 4, S 8$, and $S 12$, which refer to the conditions of high elimination rate of the parent compound, high metabolite formation rate, and first-pass effect with leads to formation of the metabolite under study, respectively.

Similar conclusions can also be observed in cases when either parent drug or metabolite exhibit tissue distribution. Figures 6(A1-A3) show the results when $\mathrm{P}$ obeys two-compartment disposition kinetics in case of the three types ( $a$, $b, c)$ of transfer-rate between the central and the peripheral compartment. In pharmacokinetic scenarios $S 4$ and $S 8$ the discrepancy in the relative permissiveness of $\mathrm{P}$ and $\mathrm{M}$ is now more obvious. However, as the $F_{T} / F_{R}$ ratio tends towards 1.25 , almost all scenarios represent, in a variant degree, a significant difference in the performance of $\mathrm{P}$ and $\mathrm{M}$. It is worth-mentioned that the differences in the performance between parent drug and metabolite data are more obvious in case of the $b$ type of transfer i.e., when the parent compound distributes rapidly to the tissues and the arrival at the central compartment follows slow kinetic rate.

In the same vein, Figures 6(B1-B3) illustrate the results in cases where metabolites exhibit peripheral distribution. These plots are in accordance with the results presented above when the parent compound followed two-compartment kinetics (Figure 6A). Nevertheless, for the $b$ type of transfer between the central and peripheral compartment, the difference in the performances of $\mathrm{P}$ and $\mathrm{M}$ is now milder (Figure 6A2 versus 6B2). 


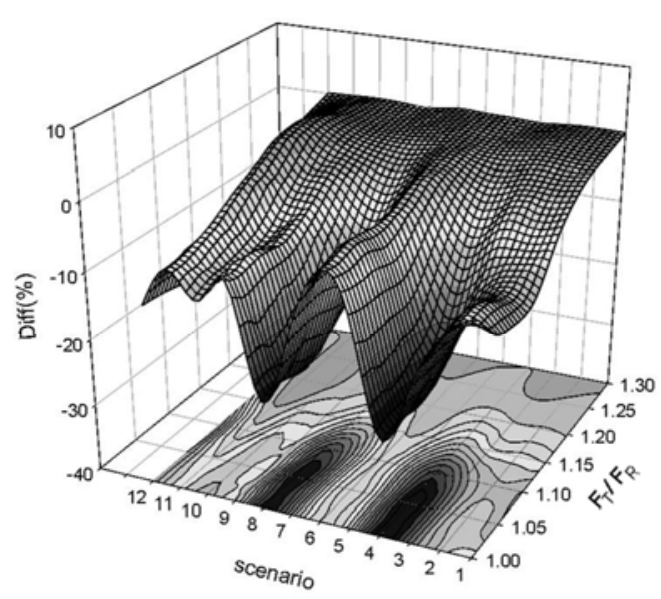

A1

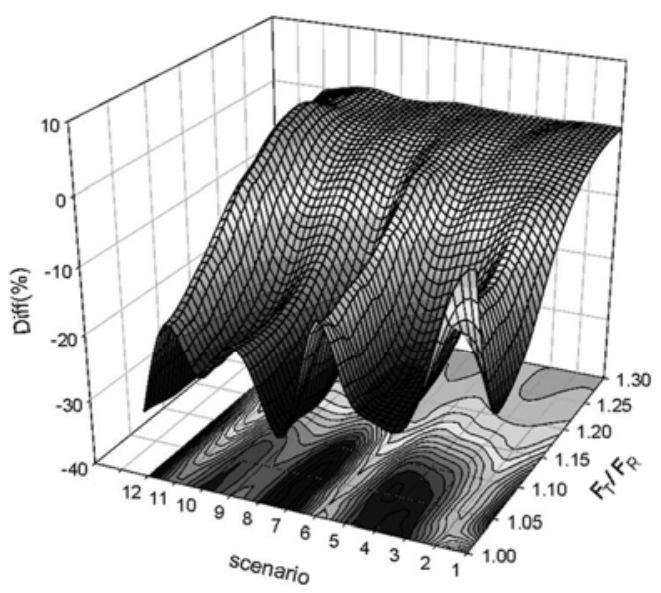

A2

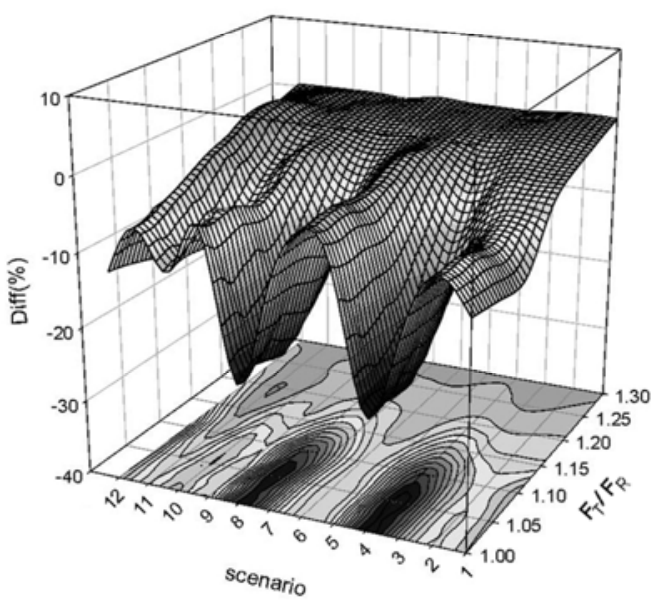

A3

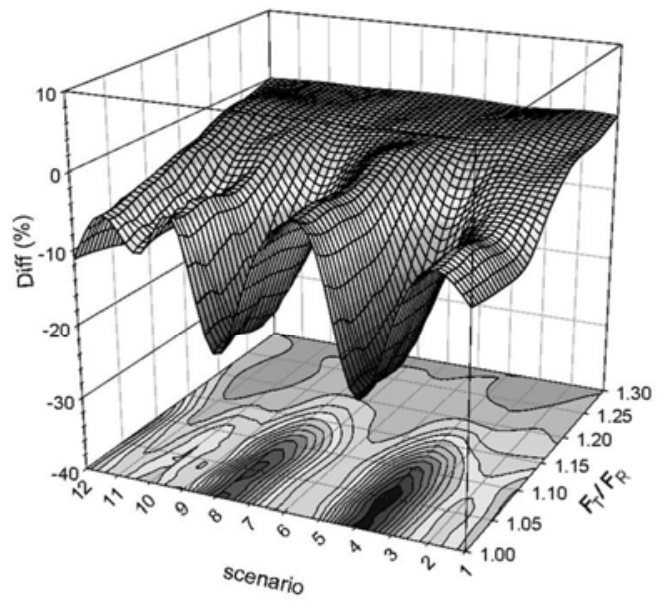

B1

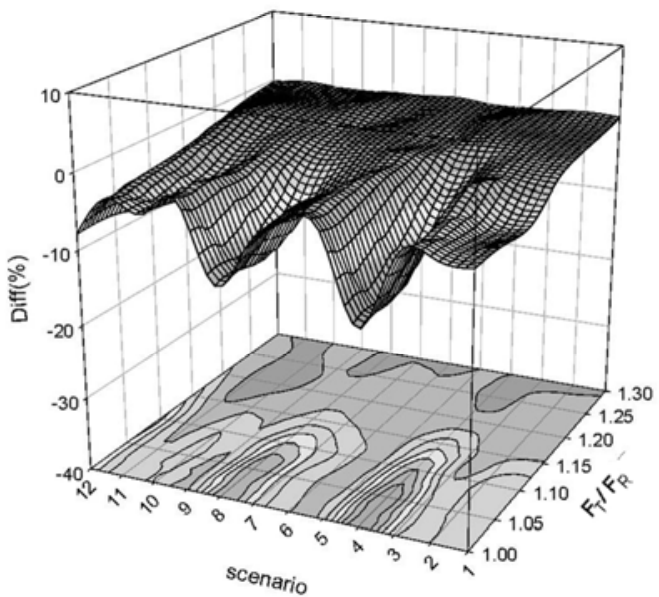

B2

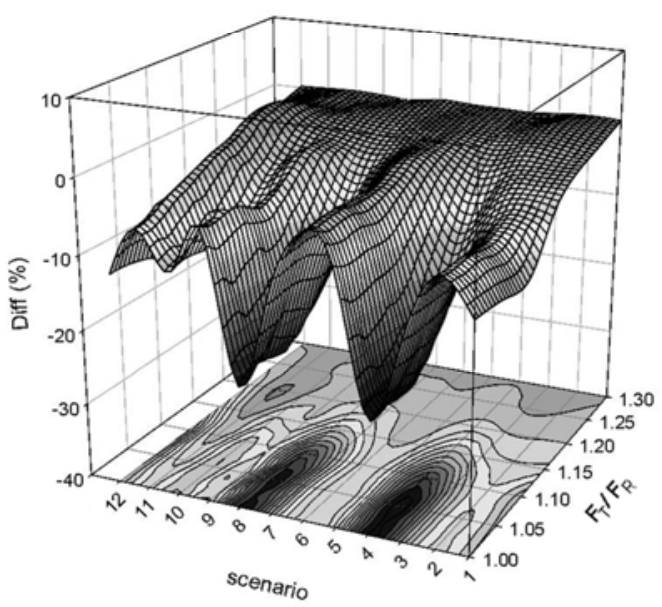

B3

Figure 4. Three dimensional power curves illustrating the difference (Diff $\%)$ in $\%$ acceptance of bioequivalence for $C_{\text {max }}$ between parent drug and metabolite at various scenarios and $F_{T} / F_{R}$ ratios in case of two-compartment model. Plots: A1-A3 parent drug exhibits tissue distribution, B1-B3 metabolite exhibits tissue distribution. Depending on the relative transfer rate between the central compartment and the tissues, three different types of kinetics are distinguished (see Table 1). Diff $\%$ is estimated by subtracting the \% acceptance of metabolite from that of the parent drug. Under each condition, a number of 5,000 studies are simulated. 


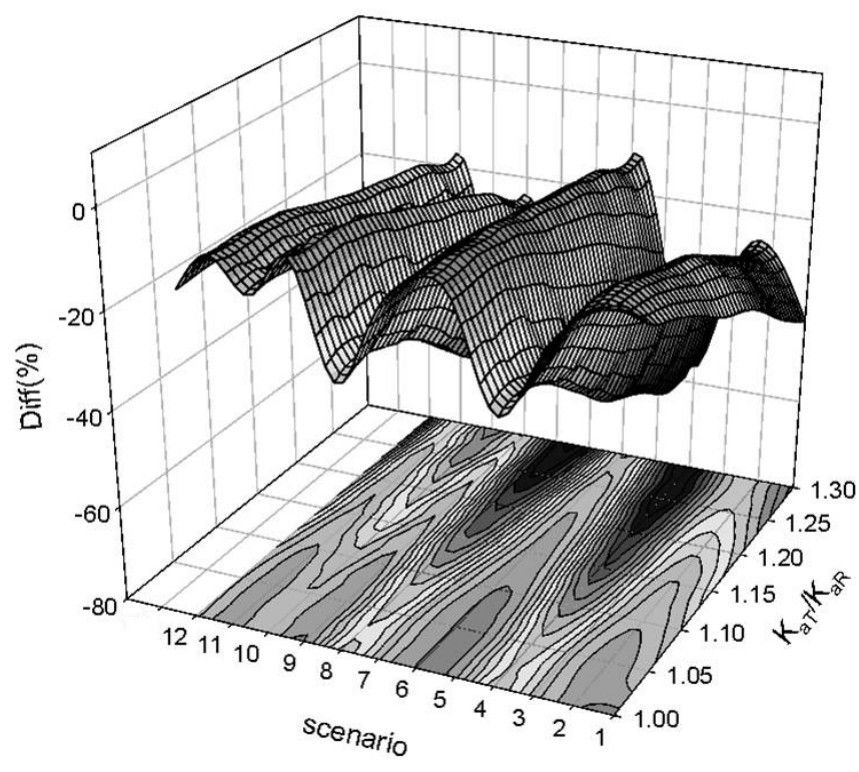

Figure 5. Three dimensional power curves illustrating the difference (Diff $\%$ ) in $\%$ acceptance of bioequivalence for $C_{\max }$ between parent drug and metabolite at various scenarios and $K_{a T} / K_{a R}$ ratios in case of one-compartment model. Diff $\%$ is estimated by subtracting the $\%$ acceptance of metabolite from that of the parent drug. Under each condition, a number of 5,000 studies are simulated.

The opposite behaviour is obtained for the $c$ type of transfer; in such cases, namely, when the metabolite undergoes tissue distribution and its departure to the periphery as well as its arrival at the central compartment occur with the same kinetic rate, the discrepancy in the performance of $\mathrm{P}$ and $\mathrm{M}$ is becoming more evident (Figure 6A3 versus $6 \mathrm{~B} 3$ ).

\section{DISCUSSION}

In this study several pharmacokinetic scenarios were simulated in order to compare the performance of the parent drug and metabolite in regard to their ability to declare bioequivalence. In all cases, $A U C$ and $C_{\max }$ were used as bioequivalence measures, while the assessment of bioequivalence was evaluated with the classic $(0.80-1.25)$ BE limits. The application of three dimensional plots, where the $\%$ difference in acceptances (Diff\%) of bioequivalence between parent drug and metabolite is plotted against $K_{a T} / K_{a R} \quad\left(\right.$ or $\left.F_{T} / F_{R}\right)$ and the pharmacokinetic scenario, allowed a global overview and comparison of the results. This kind of illustration also assisted the extraction of the similarities and discrepancies among the various models and conditions.

\section{Area Under the Curve (AUC)}

In the majority of scenarios examined for $A U C$, the probability of concluding bioequivalence was almost the same for the parent drug and the metabolite regardless of the pharmacokinetic scenario and geometric mean ratio. Therefore, no clear advantage, appears for the use of metabolites in place of parent drugs. In such cases, incorporation of metabolites in $\mathrm{BE}$ assessment can only be justified for such reasons that are already quoted in the guidelines, namely analytical purposes etc $(5,6)$.

\section{Peak Plasma Concentration $\left(C_{\max }\right)$}

A more complex behaviour was observed for $C_{\max }$ for the specific pharmacokinetic scenarios and models analyzed in this study. In most of the cases, the probability of concluding bioequivalence was higher for the metabolite than the parent drug. This attribute was more evident in situations where parent drug exhibited high elimination rate and/or the formation of the metabolite occurred rapidly. A global inspection of the results for $C_{\max }$ reveals that for the same pharmacokinetic scenario qualitatively similar results were obtained for $\mathrm{P}$ and $\mathrm{M}$ irrespective of the model (i.e., $1 C, 2 C P$ or $2 C M$ ). 


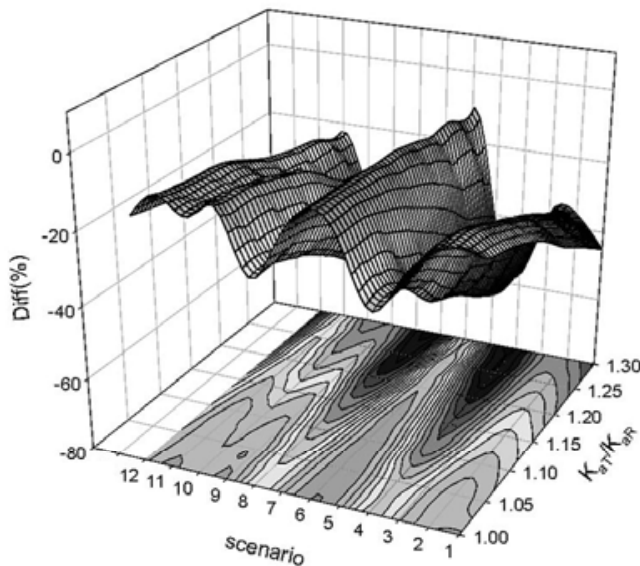

A1

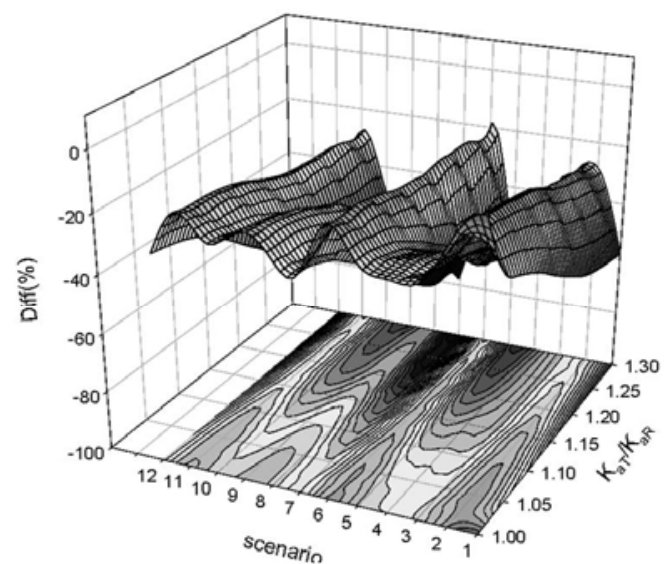

A2

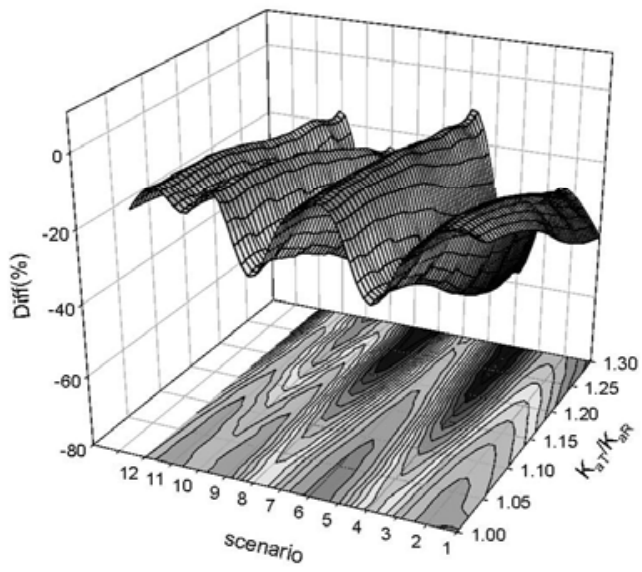

A3

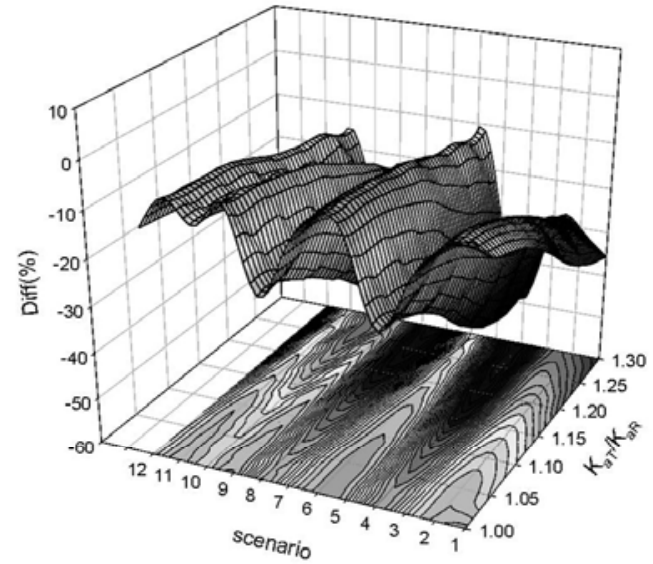

B1

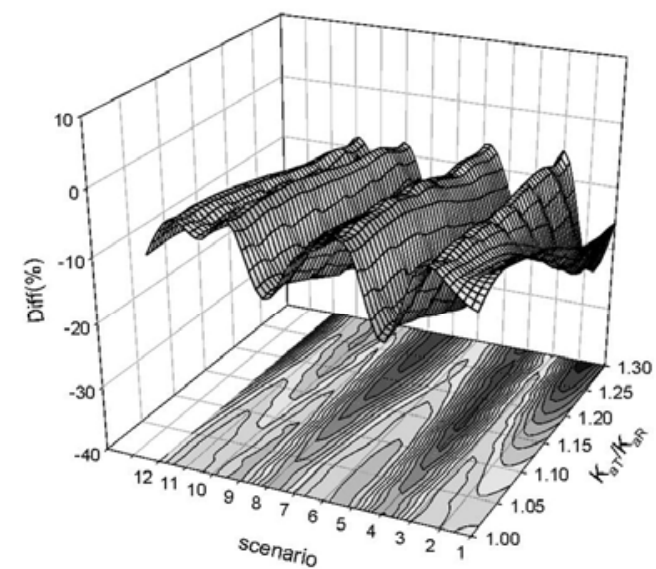

B2

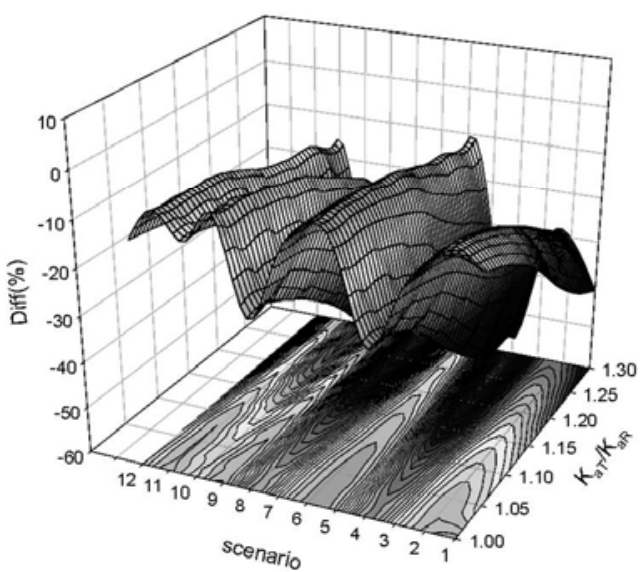

B3

Figure 6. Three dimensional power curves illustrating the difference (Diff $\%$ ) in \% acceptance of bioequivalence of $C_{\max }$ between parent drug and metabolite at various scenarios and $K_{a T} / K_{a R}$ ratios in case of two-compartment model. Plots: A1-A3 parent drug exhibits tissue distribution, B1-B3 metabolite exhibits tissue distribution. Depending on the relative transfer rate between the central compartment and the tissues, three different types of kinetics are distinguished (see Table 1). Diff \% is estimated by subtracting the \% acceptance of metabolite from that of the parent drug. Under each condition, a number of 5,000 studies are simulated. 
In cases of the $b$ type of transfer for metabolite (i.e., two-compartment disposition kinetics with very high departure rate from the central compartment and very slow arrival rate at the central compartment) the differences in the performance were fading. Exactly the opposite behaviour was observed when the parent drug followed two-compartment model (2CP model) kinetics with the $b$ type of transfer. In this case, the discrepancy of the performance between $\mathrm{P}$ and $M$ was enhanced.

\section{The Role of Extent of Absorption}

Greater percentage of $\mathrm{BE}$ acceptance for metabolite's $C_{\max }$ than parent drug's $C_{\max }$ was observed when the $F_{T} / F_{R}$ ratio was equal to unity, namely when $\mathrm{T}$ and $\mathrm{R}$ products are truly bioequivalent (Figures 3 and 4). Undoubtedly, this is a desired performance. As $F_{T} / F_{R}$ was increasing this difference was shrinking and when $F_{T} / F_{R}=1.25$ both $\mathrm{P}$ and $\mathrm{M}$ exhibited similar performance. Therefore, it seems that in cases where two drug products do not significantly differ in their extent of absorption, the use of metabolite data offers an advantage. It leads to higher percentages of acceptance when the two drug products are bioequivalent, while it maintains the same ability to declare $\mathrm{BE}$ (as parent drug data) when the two drug products do differ.

\section{The Role of Rate of Absorption - Sensitivity Analysis}

However, when the performance of $C_{\max }$ was tested over a variety of $K_{a T} / K_{a R}$ values, a much more complex behaviour was observed (Figures 5 and 6). At $K_{a T} / K_{a R}$ ratios equal to unity, metabolite data led to higher $\%$ acceptance of $\mathrm{BE}$ than parent drug data. This finding is in accordance to that described earlier for the $F_{T} / F_{R}$ ratios. Nevertheless, as $K_{a T} / K_{a R}$ increases, the discrepancy between $\mathrm{P}$ and $\mathrm{M}$ data becomes even greater (Figures 5 and 6). It seems that at $K_{a T} / K_{a R}=1$ metabolite data show a more desired performance. But, a clear answer cannot be simply given when the Ka values of the $\mathrm{T}$ and $\mathrm{R}$ products differ by $25 \%$ (i.e., when $K_{a T} / K_{a R}=1.25$ ).

Therefore, it was our aim to further examine the performance of $C_{\max }$ in cases where the $K_{a T} / K_{a R}$ ratio varies. In order to do so, an analysis of the relative sensitivity of $\mathrm{P}$ and $\mathrm{M}$ to changes of $K_{a T} / K_{a R}$ was made. This sensitivity analysis was performed according to the methodology described in the "Methods" section. According to the results of sensitivity analysis (Table 5), the ratios of the relative sensitivity are in all cases lower than unity. In certain cases, the sensitivity ratio was found to be quite small (e.g., 0.174, 0.178 etc), whereas there were situations in which both moieties exhibit almost similar sensitivity (e.g., $0.920,0.880$ etc). In other words, $C_{\max }$ data for parent drug appear to generally be more sensitive to detect differences in the absorption rate of the drug. The latter is in accordance with the general consensus on the use of parent drug data $(5,6)$.

The sensitivity analysis, however, went a step beyond and examined the relative sensitivity of $\mathrm{P}$ and $M$ data in regard to variability. Not surprisingly, metabolite data appear to be less sensitive to variability (Table 6). Similarly, there were pharmacokinetic scenarios where this difference was more evident (like in scenarios $S 4$, $S 8$ and $S 12$ ) and other cases (such as scenarios $S 6$ and S11) where parent drug and metabolite exhibited similar performance. According to Eq.1, both, high sensitivity to $K_{a T} / K_{a R}$ and to variability contribute to the ability of the moiety to declare bioequivalence. Therefore, the higher percentage of $\mathrm{BE}$ acceptance for the metabolite should be attributed to its lower overall sensitivity to conceive changes in both $K_{a T} / K_{a R}$ and variability, eventhough, the sole contribution of each factor $\left(K_{a T} / K_{a R}\right.$, variability) cannot be identified.

Overall, several recommendations can be made for $C_{\max }$ (Table 7). When the $K_{a T} / K_{a R}$ ratio of the two drug products is close to unity, metabolite data may be preferred. At these situations, the effect of sensitivity to $P K$ parameters is minimized (since the $K_{a T} / K_{a T}$ ratio is close to 1). Therefore the lower overall sensitivity can simply be ascribed to their lower sensitivity to variability. Depending on the pharmacokinetic scenario, this behaviour is more or less evident. As $\mathrm{T}$ and $\mathrm{R}$ formulations start to differ in their $K_{a}$ values, parent drug and metabolite data exhibit a more discriminant behaviour. In such cases, metabolite data become much more permissive in declaring BE. However, a clear conclusion on which moiety is preferred cannot be made. At these situations, parent drug data offer the advantage of being able to detect better the differences in the absorption rate of the two drugs. However, their ability is counterbalanced by the increased sensitivity of parent drug to variability of the data. 
Table 5. Relative sensitivity values (metabolite versus parent drug) for $C_{\max }$, in response to changes of rate of absorption. Each value was calculated as the ratio of the slopes (metabolite/parent drug i.e., slope $_{M} /$ slope $_{P}$ ) which describe the linear relationship between $G M R$ and $K_{a T} / K_{a R}$ (see paragraph "Sensitivity" in "Methods" section). Values lower than unity imply that metabolite data are less sensitive to detect differences in rate of absorption.

\begin{tabular}{cccccccc}
\hline \multirow{2}{*}{ Scenario } & \multicolumn{5}{c}{ Relative sensitivity to rate of absorption (slope $\mathrm{M}_{\text {/slope }}$ ) } \\
& 1-comp & \multicolumn{3}{c}{ 2-comp P } & \multicolumn{3}{c}{ 2-comp M } \\
\cline { 2 - 8 } & & a & $b$ & $c$ & $a$ & $b$ & $c$ \\
\cline { 2 - 7 } S1 & 0.363 & 0.389 & 0.411 & 0.383 & 0.499 & 0.793 & 0.389 \\
S2 & 0.207 & 0.187 & 0.349 & 0.237 & 0.233 & 0.509 & 0.243 \\
S3 & 0.616 & 0.590 & 0.588 & 0.623 & 0.669 & 0.922 & 0.631 \\
S4 & 0.415 & 0.416 & 0.465 & 0.419 & 0.486 & 0.732 & 0.425 \\
S5 & 0.399 & 0.364 & 0.417 & 0.409 & 0.429 & 0.798 & 0.419 \\
S6 & 0.849 & 0.880 & 0.746 & 0.852 & 0.855 & 0.920 & 0.853 \\
S7 & 0.207 & 0.174 & 0.178 & 0.218 & 0.331 & 0.753 & 0.229 \\
S8 & 0.471 & 0.476 & 0.511 & 0.477 & 0.555 & 0.760 & 0.485 \\
S9 & 0.358 & 0.356 & 0.429 & 0.369 & 0.432 & 0.836 & 0.377 \\
S10 & 0.466 & 0.509 & 0.458 & 0.479 & 0.617 & 0.829 & 0.486 \\
S11 & 0.751 & 0.697 & 0.616 & 0.757 & 0.791 & 0.874 & 0.768 \\
S12 & 0.299 & 0.321 & 0.284 & 0.310 & 0.442 & 0.712 & 0.318 \\
\hline
\end{tabular}

Table 6. Relative sensitivity values (metabolite versus parent drug) for $C_{\max }$, in response to variability. Each value was calculated as the ratio (parent drug versus metabolite) of the two residual variabilities derived from ANOVA analysis, when the $K_{a T} / K_{a R}$ ratio was equal to 1.25 (see paragraph "Sensitivity" in "Methods" section). Values lower than unity imply that metabolite data are less influenced by variability.

\begin{tabular}{cccccccc}
\hline \multirow{2}{*}{ Scenario } & \multicolumn{3}{c}{ Relative Sensitivity to variability $\left(C V w_{N} / C V w_{P}\right.$ at $\left.K_{a} / K_{a R}=1.25\right)$} \\
& 1-comp & \multicolumn{3}{c}{ 2-comp P } & \multicolumn{3}{c}{ 2-comp M } \\
\cline { 2 - 8 } S1 & & $a$ & $b$ & $c$ & $a$ & $b$ & $c$ \\
\cline { 2 - 7 } S2 & 0.860 & 0.853 & 0.772 & 0.862 & 0.877 & 0.943 & 0.864 \\
S3 & 0.891 & 0.872 & 0.887 & 0.893 & 0.898 & 0.921 & 0.896 \\
S4 & 0.860 & 0.841 & 0.812 & 0.860 & 0.874 & 0.966 & 0.863 \\
S5 & 0.772 & 0.770 & 0.787 & 0.771 & 0.792 & 0.882 & 0.773 \\
S6 & 0.894 & 0.865 & 0.785 & 0.892 & 0.900 & 0.959 & 0.896 \\
S7 & 0.962 & 0.961 & 0.899 & 0.961 & 0.961 & 0.975 & 0.961 \\
S8 & 0.839 & 0.833 & 0.719 & 0.838 & 0.853 & 0.933 & 0.841 \\
S9 & 0.792 & 0.792 & 0.807 & 0.791 & 0.817 & 0.900 & 0.794 \\
S10 & 0.919 & 0.881 & 0.798 & 0.916 & 0.923 & 0.972 & 0.920 \\
S11 & 0.885 & 0.880 & 0.800 & 0.884 & 0.905 & 0.954 & 0.886 \\
S12 & 0.948 & 0.929 & 0.884 & 0.949 & 0.956 & 0.974 & 0.951 \\
\hline
\end{tabular}




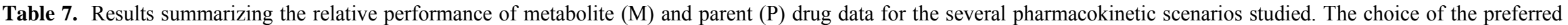
analyte depends on the $\%$ acceptance of bioequivalence, the relative sensitivity to changes of $F_{T} / F_{R}, K_{a T} / K_{a R}$, and variability.

\begin{tabular}{|c|c|c|c|c|c|c|c|}
\hline \multirow{2}{*}{\multicolumn{2}{|c|}{ Change }} & \multirow[b]{2}{*}{ PK scenario } & \multirow{2}{*}{$\begin{array}{l}\text { \% Acceptance of } \\
\text { Bioequivalence }^{1}\end{array}$} & \multicolumn{2}{|c|}{ Sensitivity } & \multirow{2}{*}{$\begin{array}{c}\text { Possibly } \\
\text { Preferred } \\
\text { Analyte } \\
\end{array}$} & \multirow[b]{2}{*}{ Reason } \\
\hline & & & & $\begin{array}{c}\text { Slope } \\
F_{T} / F_{R} \text { or } K_{a T} / K_{a R}\end{array}$ & Variability & & \\
\hline \multicolumn{8}{|c|}{ AUC } \\
\hline \multirow{2}{*}{$F_{T} / F_{R}$} & $F_{T} / F_{R}=1.00$ & $\mathrm{~S} 1-\mathrm{S} 12$ & $\mathrm{M}=\mathrm{P}$ & $\mathrm{M}=\mathrm{P}$ & $\mathrm{M}=\mathrm{P}$ & $\mathrm{M}$ or $\mathrm{P}$ & - \\
\hline & $F_{T} / F_{R}=1.25$ & $\mathrm{~S} 1-\mathrm{S} 12$ & $\mathrm{M}=\mathrm{P}$ & $\mathrm{M}=\mathrm{P}$ & $\mathrm{M}=\mathrm{P}$ & $\mathrm{M}$ or $\mathrm{P}$ & - \\
\hline$K_{a T} / K_{a R}$ & $K_{a T} / K_{a R}=1.00$ & $\mathrm{~S} 1-\mathrm{S} 12$ & $\mathrm{M}=\mathrm{P}$ & $\mathrm{M}=\mathrm{P}$ & $\mathrm{M}=\mathrm{P}$ & $\mathrm{M}$ or $\mathrm{P}$ & - \\
\hline \multicolumn{8}{|c|}{$C_{\max }$} \\
\hline \multirow{4}{*}{$F_{T} / F_{R}$} & & $\mathrm{~S} 4, \mathrm{~S} 8$ & $\mathrm{M}>>\mathrm{P}$ & $\mathrm{M}=\mathrm{P}$ & $\mathrm{M}<<\mathrm{P}$ & M & Reduced Variability of $\mathrm{M}$ data \\
\hline & $F_{T} / F_{R}=1.00$ & $\mathrm{~S} 1, \mathrm{~S} 3, \mathrm{~S} 7, \mathrm{~S} 10, \mathrm{~S} 12$ & $\mathrm{M}>\mathrm{P}$ & $\mathrm{M}=\mathrm{P}$ & $\mathrm{M}<\mathrm{P}$ & $\mathrm{M}$ & Reduced Variability of $\mathrm{M}$ data \\
\hline & & $\mathrm{S} 2, \mathrm{~S} 5, \mathrm{~S} 6, \mathrm{~S} 9, \mathrm{~S} 11$ & $\mathrm{M} \approx \mathrm{P}$ & $\mathrm{M}=\mathrm{P}$ & $\mathrm{M} \approx \mathrm{P}$ & $\mathrm{M}$ or $\mathrm{P}$ & - \\
\hline & $F_{T} / F_{R}=1.25$ & $\mathrm{~S} 1-\mathrm{S} 12$ & $\mathrm{M}=\mathrm{P}$ & $\mathrm{M}=\mathrm{P}$ & $\mathrm{M}=\mathrm{P}$ & $\mathrm{M}$ or $\mathrm{P}$ & - \\
\hline \multirow{3}{*}{$K_{a T} / K_{a R}$} & & $\mathrm{~S} 4, \mathrm{~S} 8, \mathrm{~S} 12$ & $\mathrm{M}>\mathrm{P}$ & $\mathrm{M}=\mathrm{P}$ & $\mathrm{M}<\mathrm{P}$ & M & Reduced Variability of $\mathrm{M}$ data \\
\hline & & S6, S9 & $\mathrm{M} \approx \mathrm{P}$ & $\mathrm{M}=\mathrm{P}$ & $\mathrm{M} \approx \mathrm{P}$ & $\mathrm{M}$ or $\mathrm{P}$ & - \\
\hline & $K_{a T} / K_{a R}=1.25$ & $\mathrm{~S} 1-\mathrm{S} 12$ & $\mathrm{M}>\mathrm{P}$ & $\mathrm{M}<\mathrm{P}^{2}$ & $\mathrm{M}<\mathrm{P}$ & $?^{3}$ & $\begin{array}{l}\text { Depends on relative sensitivity } \\
\text { to PK parameters and variability }\end{array}$ \\
\hline
\end{tabular}

${ }^{1}$ Based on the total of the simulated scenarios.

${ }^{2}$ The magnitude of the difference depends on the scenario.

${ }^{3}$ A robust conclusion cannot be made since the decision depends on the relative contribution of $K_{a T} / K_{a R}$ changes and variability to overall sensitivity. 
Nevertheless, it should not disregarded the fact that even in these situations (i.e., with a $25 \%$ difference in $K_{a}$ values) the $\mathrm{T}$ and $\mathrm{R}$ products can still be bioequivalent since their GMR ratio is lower than 1.25 .

\section{CONCLUSIONS}

Aim of this study was to examine the conditions where the behaviour of the parent drug differentiates from that of the metabolite assuming a variety of pharmacokinetic conditions. Even though the simulated pharmacokinetic scenarios of this work were not alike with those already presented in other published studies, the basic conclusions derived from this analysis were consistent with the findings of the other studies (21, 23-27).

Since, the bioequivalence decision depends on the ability of the measured moiety to identify differences in the responses between the test and reference formulation, the analyte of choice would be the one which would reflects more precisely the differences in the extent and the rate of absorption. This implies that the analyte which transfers the information of bioequivalence with higher sensitivity and lower variability will be preferred.

In case of $A U C$, both $\mathrm{P}$ and $\mathrm{M}$ data exhibit the same performance and can lead to the same conclusions. Therefore, none of them offers a clear advantage over the other regarding their use in BE studies. Presumably, this finding does not imply that other criteria (such as analytical properties) may not suggest the application of a specific moiety. For $C_{\max }$, a more complex behaviour is observed. In general, metabolite $C_{\max }$ data seem to be preferable when either the $T$ and $\mathrm{R}$ products are truly bioequivalent or the two formulations differ in their extent of absorption. Parent drug data also appeared to be more sensitive to detect differences in the rate of absorption. However, in such cases, the information is much influenced by the increased variability which accompanies parent drug data.

\section{NOVELTY OF THE WORK}

To investigate the role of metabolites in bioequivalence assessment by defining the criteria for the selection of the "preferred analyte" lying on a sensitivity basis. The preferred analyte identifies the differences in the responses, between the two formulations, with higher sensitivity and lower variability. In case of $A U C$, parent drug and metabolite data exhibit similar behaviour. Metabolite $C_{\max }$ data are preferable when the two drug products are truly bioequivalent or differ only in their extent of absorption. The greater sensitivity of parent drug data to detect differences in the rate of absorption is counterbalanced by their high sensitivity to variability.

\section{KEY WORDS}

Bioequivalence; Metabolite; Sensitivity; Pharmacokinetic simulations

\section{ABBREVIATIONS}

$A U C$, area under the concentration-time curve; $\mathrm{BE}$, Bioequivalence; $C$, concentration; $C_{\max }$, the peak concentration of a concentration-time curve; $C V_{W}$, intra-individual variability; GI, gastrointestinal tract; GMR, geometric mean ratio of the bioavailability measures; $\mathrm{M}$, metabolite; $\mathrm{P}$, parent drug; PK, pharmacokinetics; $t$, time; $90 \%$ CI, $90 \%$ confidence interval; $1 C$, onecompartment model; 2CM, two-compartment model for the metabolite; $2 C P$, two-compartment model for the parent drug;

\section{REFERENCES}

1. Food and Drug Administration. Bioavailability and Bioequivalence Studies for Orally Administered Drug Products. General Consideration, Center for Drug Evaluation and Research (CDER), Rockville, MD, 2000.

2. Houston, J.B. Importance of metabolites in bioequivalence, in McGilveray JJ: Dighe SV: French IW: Midha KK: Skelly JP (eds.), Proceedings Bio-International 89: Issues in the evaluation of bioavailability data. Toronto, Canada, pp 99-100, 1989.

3. Tucker, G., Rostami, A., Jackson, P. Metabolite measurement in bioequivalence studies: Theoretical considerations, in Midha KK: Blume $\mathrm{H}$ (eds.), Bio-International: Bioavailability, Bioequivalence and Pharmacokinetics. International Conference of FIP. "BioInternational'92", Bad Homburg, Germany, Medpharm Scientific Publishers, Stuttgart, pp 163-170, 1993.

4. A.J. Jackson, G. Robbie, P. Marroum. Metabolites and bioequivalence: past and present. Clin Pharmacokinet, 43:655-672, 2004.

5. K. Midha, M. Rawson, and J. Hubbard. The Role of Metabolites in Bioequivalence. Pharm Res, 21:1331-1344, 2004. 
6. K. Midha, V. Shah, G. Singh, R. Patnaik. Conference report: Bio-International 2005. J Pharm Sci, 96:747-754, 2007.

7. Food and Drug Administration. Guidance for Industry. Bioavailability and Bioequivalence Studies for Orally Administered Drug Products General Considerations, 2003.

8. European Agency for the Evaluation of Medicinal Products. Note for Guidance on the Investigation of Bioavailability and Bioequivalence. Committee for Proprietary Medicinal Products (CPMP), London, 2001.

9. European Medicinal Evaluation Agency. Committee for Medicinal Products for Human Use (CHMP). Concept Paper for an Addendum to the Note for Guidance on the Investigation of Bioavailability and Bioequivalence: Evaluation of Bioequivalence of Highly Variable Drugs and Drug Products. London, 2006.

10. European Medicines Agency, Evaluation of Medicines for Human Use, CHMP efficacy working party) therapeutic subgroup on pharmacokinetics: Questions \& Answers on the Bioavailability and Bioequivalence Guideline, London, 2006.

11. I. Mahmood. Assessment of metabolites in bioequivalence studies: should bioequivalence criteria be applied on the sum of parent compound and metabolite? Int $\mathrm{J}$ Clin Pharmacol Ther, 36:540-544, 1998.

12. K. Midha, J. Hubbard, G. McKay, E. Hawes, D. Hsia. The role of metabolites in a bioequivalence study 1: loxapine, 7-hydroxyloxapine and 8hydroxyloxapine. Int $\mathrm{J}$ Clin Pharmacol Ther Toxicol, 31:177-183, 1993.

13. K. Midha, J. Hubbard, G. McKay, M. Rawson, D. Hsia D. The role of metabolites in a bioequivalence study II: amoxapine, 7hydroxyamoxapine, and 8-hydroxyamoxapine. Int J Clin Pharmacol Ther, 37:428-438, 1999.

14. V. Hutt, K. Sauter, G. Pabst, R. Bonn, E. Fritschi. Bioequivalence evaluation of the metabolites 1,2 and 1,3-glyceryl dinitrate of two different glyceryl trinitrate patches after 12-h usage in healthy volunteers. Arzneimittelforschung, 44:1317-1321, 1994.

15. W.M. Glazer, L.T. Friedhoff, S.R. Marder, and W.A. Brown. The determination of the steadystate pharmacokinetic profile of fluphenazine decanoate by gas chromatography/mass spectrometry detection. Schizophr Res, 8:111117, 1992.
16. H. Mascher, C. Kikuta, A. Millendorfer, H. Schiel, and G. Ludwig. Pharmacokinetics and bioequivalence of the main metabolites of selegiline: Desmethylselegiline, methamphetamine and amphetamine after oral administration of selegiline. Int J Clin Pharm Ther, 35:9-13, 1997.

17. E. Heinonen, M. Anttila, and A. Lammintausta. Pharmacokinetic aspects of 1-deprenyl (selegiline) and its metabolites. Clin Pharm Therap, 56:742749, 1994.

18. H. R. Kwon, P. Green, and S. Curry. Pharmacokinetics of nitroglycerin and its metabolites after administration of sustainedrelease tablets. Biopharm Drug Dispos, 13:141152, 1992.

19. E. Ezan, T. Ardouin, B. Landes, B. Flouvat, T. Hanslik, J. Legeai, and J. Grognet. Bioequivalence study of alphadihydroergocryptine utility of metabolite evaluation. Int J Clin Pharmacol Ther, 34:32-37, 1996.

20. H. Vergin, G. Mahr, R. Metz, A. Eichinger, V. Nitsche, and H. Martens H. Analysis of metabolites - a new approach to bioequivalence studies of spironolactone formulations. Int J Clin Pharmacol Ther, 35:334-340, 1997.

21. M.L. Chen, A.J. Jackson. The role of metabolites in bioequivalency assessment. I. Linear pharmacokinetics without first-pass effect. Pharm Res, 8:25-32, 1991.

22. M.L. Chen, A.J. Jackson. The role of metabolites in bioequivalency assessment. II. Drugs with linear pharmacokinetics and first-pass effect. Pharm Res, 12:700-708, 1995.

23. S. Rosenbaum and J. Lam. Bioequivalence parameters of parent drug and its first-pass metabolite: comparative sensitivity to sources of pharmacokinetic variability. Drug Dev Indust Pharm, 23:337-344, 1997.

24. A.J. Jackson. The role of metabolites in bioequivalency assessment. III. Highly variable drugs with linear kinetics and first-pass effect. Pharm Res, 17:1432-1436, 2000.

25. A.C. Braddy, A.J. Jackson. Role of metabolites for drugs that undergo nonlinear first-pass effect: impact on bioequivalency assessment using singledose simulations. J Pharm Sci, 99:515-523, 2010.

26. C. Fernández-Teruel, R. Nalda Molina, I. González-Alvarez, C. Navarro-Fontestad, A. García-Arieta, V.G. Casabó, M. Bermejo. Computer simulations of bioequivalence trials: selection of design and analyte in BCS drugs with first-pass hepatic metabolism: linear kinetics (I). Eur J Pharm Sci, 36:137-146, 2009. 
27. C. Fernández-Teruel, I. Gonzalez-Alvarez, C. Navarro-Fontestad, A. García-Arieta, M. Bermejo, V.G. Casabó. Computer simulations of bioequivalence trials: selection of design and analyte in BCS drugs with first-pass hepatic metabolism: Part II. Non-linear kinetics. Eur J Pharm Sci, 36:147-156, 2009.

28. D.J. Schuirmann. A comparison of the two onesided tests procedure and the power approach for assessing the equivalence of average bioavailability. J Pharmacokin Biopharm, 15:657680, 1987.

29. L. Tothfalusi, S. Speidl, L. Endrenyi. Exposureresponse analysis reveals that clinically important toxicity difference can exist between bioequivalent carbamazepine tablets. $\mathrm{Br} \mathrm{J}$ Clin Pharmacol, 65:110-122, 2007.

\section{APPENDIX}

\section{Systems of Ordinary Differential Equations}

Sets of differential equations were used to generate concentration-time data for both parent drug $(\mathrm{P})$ and metabolite (M).

\section{One-compartment model (Figure 1A of the manuscript)}

GI: Parent drug

$$
\frac{d M_{G I}}{d t}=-K_{a} \cdot M_{G I}
$$

Central compartment: Parent drug

Central compartment: Metabolite

$$
\frac{d C_{P}}{d t}=K_{a} \cdot \frac{M_{G I}}{V_{1, P}}-C_{P} \cdot\left(K_{f}+K_{e l, P}\right)
$$

$$
\frac{d C_{M}}{d t}=K_{f} \cdot \frac{C_{p} V_{1, p}}{V_{1, M}}-K_{e l, M} \cdot C_{M}
$$

Initial conditions:

$$
\begin{aligned}
& C_{P}(0)=C_{M}(0)=0 \\
& M_{G I}(0)=F \cdot \text { Dose }
\end{aligned}
$$

\section{Two-compartment disposition kinetics for the parent drug with first-pass effect (Figure $1 \mathrm{H}$ of the} manuscript)

GI: Parent drug

$$
\frac{d M_{G I}}{d t}=-K_{a} \cdot M_{G I}-M M_{0} \cdot \frac{M_{G I}}{M M_{50}+M_{G I}}
$$

Central compartment: Parent drug

$$
\frac{d C_{1, P}}{d t}=K_{a} \cdot \frac{M_{G I}}{V_{1, P}}-C_{1, P} \cdot\left(K_{f}+K_{e l, P}\right)-K_{12, P} \cdot C_{1, P}+K_{21, P} \cdot \frac{M_{2, P}}{V_{1, P}}
$$

Central compartment: Metabolite

$$
\frac{d C_{M}}{d t}=K_{f} \cdot \frac{C_{1, P} \cdot V_{1, P}}{V_{1, M}}-K_{e l, M} \cdot C_{M}+\frac{M M_{0}}{V_{1, M}} \cdot \frac{M_{G I}}{M M_{50}+M_{G I}}
$$

Peripheral compartment: Parent drug

$$
\frac{d M_{2, P}}{d t}=K_{12, P} \cdot C_{1, P} \cdot V_{1, P}-K_{21, P} \cdot M_{2, P}
$$

Initial conditions: 


$$
\begin{aligned}
& C_{1, P}(0)=C_{M}(0)=C_{2, P}(0)=0 \\
& M_{G I}(0)=F \cdot \text { Dose }
\end{aligned}
$$

Key: $F$, the bioavailable fraction of dose; $K_{a}$, absorption rate constant, $K_{e l, i}$, elimination rate constant; $K_{f}$, first-order formation rate constant of the metabolite; $V_{l, i}$, volume of distribution in central compartment; $M M_{0}$, maximum formation rate for $1^{\text {st }}$ pass effect; $M M_{50}$, Michaelis-Menten constant for $1^{\text {st }}$ pass effect; $K_{12, i}$ central compartment to tissue transfer rate constant; $K_{21, i}$ tissue to central compartment transfer rate constant; $M_{G I}$, amount of drug absorbed; $M_{i}$, amount of the moiety $i$ in the compartment; $C_{i}$, concentration of the moiety $i$ in the compartment. In all cases the index $i$ refers to either P or M. 